\title{
Neuro-Fuzzy Approach to Heart Rate Variability Analysis
}

\author{
Hoang ChuDuc, Thuan NguyenDuc, and Trung LaiHuuPhuong
}

\begin{abstract}
Heart rate variability (HRV) analysis attempts to assess cardiac autonomic regulation through quantification of sinus rhythm variability. The sinus rhythm times series is derived from the QRS to QRS (RR) interval sequence of the electrocardiogram (ECG), by extracting only normal sinus to normal sinus (NN) interbeat intervals. Relatively high frequency variations in sinus rhythm reflect parasympathetic (vagal) modulation, and slower variations reflect a combination of both parasympathetic and sympathetic modulation and non-autonomic factors. The paper focuses on the Neuro-fuzzy system. It is used to recognize the HRV signals for diagnosis by extract the QRST zone of ECG signals using Discrete Cosine Transform (DCT). The result is exciting as much as we have used only one of ECG lead to records input data, while the current diagnosis approaches require the set of 12 lead ECG signals!
\end{abstract}

Index Terms-Heart rate variability, neuro-fuzzy, discrete cosine transform.

\section{INTRODUCTION}

Traditional heart rate variability (HRV) measures are usually divided into two broad categories: time domain measures and frequency domain measures [1]. The time domain heart rate variability statistics commonly calculated are defined in Table I.

TABLE I: COMMONLY USED TIME-DOMAIN MEASURES

\begin{tabular}{|c|c|}
\hline Name & Descriptions \\
\hline SDNN & Standard deviation of all NN intervals \\
\hline SDANN & $\begin{array}{l}\text { Standard deviation of the averages of } \mathrm{NN} \text { intervals in all } \\
5 \text {-minute segments of a } 24 \text {-hour recording }\end{array}$ \\
\hline SDNNIDX & $\begin{array}{l}\text { Mean of the standard deviations of } \mathrm{NN} \text { intervals in all } \\
5 \text {-minute segments of a } 24 \text {-hour recording }\end{array}$ \\
\hline rMSSD & $\begin{array}{l}\text { Square root of the mean of the squares of differences } \\
\text { between adjacent NN intervals }\end{array}$ \\
\hline pNN50 & $\begin{array}{l}\text { Percentage of differences between adjacent } \mathrm{NN} \text { intervals } \\
\text { that are greater than } 50 \mathrm{~ms} \text {; a member of the larger } \mathrm{pNNx} \\
\text { family }\end{array}$ \\
\hline AVNN & Average of all NN intervals \\
\hline
\end{tabular}

Note, however, that computing pNNx with $x<50 \mathrm{~ms}$ in both long- and short-term recordings may provide a more robust index of fluctuations due to vagal tone than the standard pNN50 statistic [2]. Commonly used frequency domain measures are defined in Table II. The low frequency band $(0.04-0.15 \mathrm{~Hz})$ includes physiologic oscillations

Manuscript received February 1, 2013; revised April 2, 2013.

Hoang ChuDuc and Thuan NguyenDuc are with Department of Electronics Technology and Biomedical Engineering, School of Electronics and Telecommunications, Hanoi University of Scicence and Technology, Hanoi, Vietnam (e-mail: hoang.chuduc@hust.edu.vn, thuan.nguyenduc @ hust.edu.vn).

Trung LaiHuuPhuong is with Center of Electronics and Biomedical, Hanoi University of Scicence and Technology, Hanoi, Vietnam (e-mail: trung.laihuuphuong@hust.edu.vn). associated with baroreceptor reflexes and the high frequency band $(0.15-0.40 \mathrm{~Hz})$ encompasses respiratory sinus arrhythmia. The powers in these bands have been used to provide indexes of autonomic function. Such measures must be interpreted with caution, however. As noted, oscillations in the "low" frequency bands appear to be mediated by parasympathetic and sympathetic components, while the "high" frequency power is mediated exclusively by the vagus.

TABLE II: COMMONLY USED FREQUENCY-DOMAIN MEASURES

\begin{tabular}{ll}
\hline \hline Name & Descriptions \\
\hline TOTPWR & Total spectral power of all NN intervals up to $0.04 \mathrm{~Hz}$ \\
ULF & Total spectral power of all NN intervals up to $0.003 \mathrm{~Hz}$ \\
& Total spectral power of all NN intervals between 0.003 \\
VLF & and $0.04 \mathrm{~Hz}$ \\
Total spectral power of all NN intervals between 0.04 \\
and $0.15 \mathrm{~Hz}$.
\end{tabular}

Recently, there are many approaches involving techniques for computer processing of 12 lead electrocardiograms (ECG), in order to diagnose a HRV signal. A first group of methods to interpret the ECG significance uses a morphological analysis. For example, myocardial ischemia may produce a flat or inverted $\mathrm{T}$ wave, that is classical narrow and symmetrical. A second group of techniques for computer analysis of ECG uses statistical models. In a statistical model and the corresponding experimental results are presented for the classification of ECG patterns to diagnose the HRV signal. A third group of methods corresponding to neural models becomes a powerful concurrent to statistical ones for HRV signal classification [3].

The hybrid systems of fuzzy logic and neural networks [4] often referred as fuzzy neural networks represent exciting models of computational intelligence with direct applications in pattern recognition, approximation, and control. We further perform signal classification for HRV analysis using the neuro-fuzzy classifier called Neuro-fuzzy system. Neuro-fuzzy system has been obtained as a modified version of the fuzzy neural network described by Chen and Teng in [5], as identifier in control systems. We have applied this model here in an ECG recognition cascade for HRV analysis by extract HRV signal using either Principal Component Analysis (PCA) or Discrete Cosine Transform (DCT).

\section{METHODS}

\section{A. Neuro-Fuzzy System}

In the field of artificial intelligence, neuro-fuzzy refers to combinations of artificial neural networks and fuzzy logic. 
Neuro-fuzzy was proposed by J. S. R. Jang [6]. Neuro-fuzzy hybridization results in a hybrid intelligent system that synergizes these two techniques by combining the human-like reasoning style of fuzzy systems with the learning and connectionist structure of neural networks. Neuro-fuzzy hybridization is widely termed as Fuzzy Neural Network (FNN) or Neuro-Fuzzy System (NFS) in the literature. Neuro-fuzzy system (the more popular term is used henceforth) incorporates the human-like reasoning style of fuzzy systems through the use of fuzzy sets and a linguistic model consisting of a set of IF-THEN fuzzy rules. The main strength of neuro-fuzzy systems is that they are universal approximators with the ability to solicit interpretable IF-THEN rules. Neuro-fuzzy system has four-layer that described in in Fig. 1.

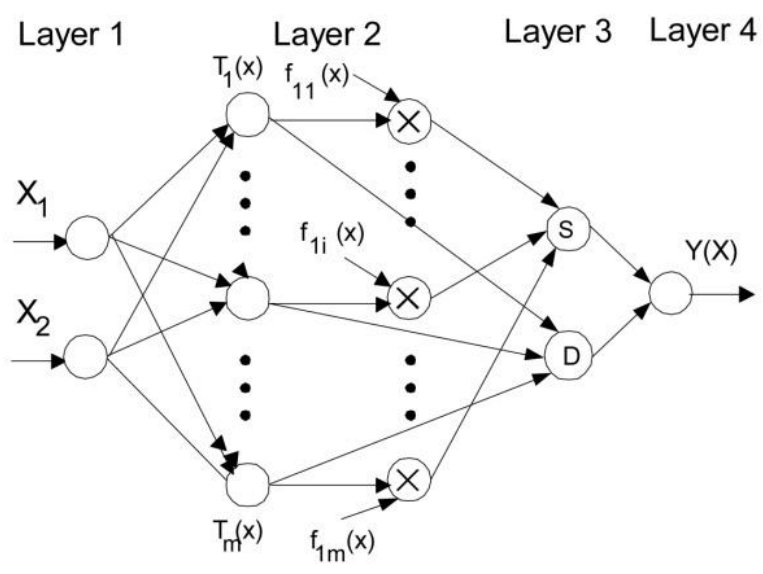

Fig. 1. Structure of neuro-fuzzy system.

The change affects only the equations of the fourth layer, while the structure diagram is similar. Its construction is based on fuzzy rules of the form:

$R j$ : If $x 1$ is $A 1 j$ and $x 2$ is $A 2 j \ldots$ and $x m$ is $A m j$, then $y 1$ is

$$
\beta 1 j, \ldots, y M \text { is } \beta M j \text {, }
$$

where $m$ is the dimension of the input vectors (number of retained features), and $\mathrm{j}$ is the rule index $(j=1 \ldots K)$. The number of output neurons (of the fourth layer) corresponds to the number of classes and it is equal to M. Neuro-fuzzy system keeps the advantages of the original fuzzy net described by Chen and Teng [7] for identification in control systems: a) its structure allows us to construct the fuzzy system rule by rule; $b$ ) if the prior knowledge of an expert is available, then we can directly add some rule nodes and term nodes; c) the number of rules do not increase exponentially with the number of inputs; d) elimination of redundant nodes rule by rule.

Each neuron performs two actions using two different functions. The first is the aggregation function gk ( ), which computes the net input

$$
\text { Netinput }=g^{k}\left(x^{k} ; W^{k}\right)
$$

where the superscript indicates the layer number $(k=1, . ., 4)$, $x^{k}$ is the input vector and $W^{k}$ is the weight vector. The second function is the nonlinear activation function $f^{k}($ ), which gives

$$
\text { Output }=O_{i}^{k}=f^{k}\left(g^{k}\right)
$$

where $O_{i}^{k}$ is the i-th output of the k-th layer.

Layer 1 is fuzzyifying inputs,

$$
\begin{gathered}
O_{1, i}=\mu A i(x), \text { for } i=1,2 \text { and } \\
O_{1, i-2}=\mu B i(y) \text { for } i=3,4,
\end{gathered}
$$

where $O$ is the output of the layer 1 , node $i$

Layer 2 calculates the firing strength of a rule,

$$
O_{2, i}=w i=\mu A i(x) \mu B i(y), i=1,2 .
$$

Layer 3 normalize firing strength of the node $i$,

$$
O_{3, i}=\overline{w_{i}}=\frac{w_{i}}{w_{1}+w_{2}}, i=1,2
$$

Layer 4 calculates the conclusions,

$$
o_{4, i}=\bar{w}_{i} f_{i}=\bar{w}\left(p_{i} x+q_{i} y+r_{i}\right),
$$

\section{B. Heart Rate Variability Analysis}

The most widely used methods can be grouped under time-domain and frequency-domain. Other methods have been proposed, such as non-linear methods.

Time-domain methods: These are based on the beat-to-beat or $\mathrm{NN}$ intervals, which are analyzed to give variables such as: SDNN, the standard deviation of NN intervals. Often calculated over a 24-hour period. SDANN, the standard deviation of the average NN intervals calculated over short periods, usually 5 minutes. SDANN is therefore a measure of changes in heart rate due to cycles longer than 5 minutes. SDNN reflects all the cyclic components responsible for variability in the period of recording, therefore it represents total variability.

Geometric methods: The series of NN intervals also can be converted into a geometric pattern such as the sample density distribution of NN interval durations, sample density distribution of differences between adjacent NN intervals, Lorenz plot of NN or RR intervals, and so forth, and a simple formula is used that judges the variability on the basis of the geometric and/or graphics properties of the resulting pattern.

Frequency-domain methods: Several methods are available. Power spectral density (PSD), using parametric or nonparametric methods, provides basic information on the power distribution across frequencies. One of the most commonly used PSD methods is the discrete Fourier transform. Methods for the calculation of PSD may be generally classified as nonparametric and parametric. In most instances, both methods provide comparable results. The advantages of the nonparametric methods are the simplicity of the algorithm used (fast Fourier transform [FFT] in most of the cases) and the high processing speed, while the advantages of parametric methods are smoother spectral components that can be distinguished independent of 
preselected frequency bands, easy post processing of the spectrum with an automatic calculation of low- and high-frequency power components with an easy identification of the central frequency of each component, and an accurate estimation of PSD even on a small number of samples on which the signal is supposed to maintain stationary. The basic disadvantage of parametric methods is the need of verification of the suitability of the chosen model and of its complexity (that is, the order of the model).

In addition to classical FFT-based methods used for the calculation of frequency parameters, a more appropriate PSD estimation method is the Lomb-Scargle (LS) period gram [10]. Analysis has shown that the LS period gram can produce a more accurate estimate of the PSD than FFT methods for typical RR data. Since the RR data is an unevenly sampled data, another advantage of the LS method is that in contrast to FFT-based methods it is able to be used without the need to resample and detrend the RR data.

A newly used HRV index, which depends on the wavelet entropy measures, is an alternative choice. The wavelet entropy measures are calculated using a three-step procedure defined in the literature. First, the wavelet packet algorithm is implemented using the Daubechies 4 (DB4) function as the mother wavelet with a scale of 7 . Once the wavelet coefficients are obtained, the energy for each coefficient are calculated as described in the literature. After calculating the normalized values of wavelet energies, which represent the relative wavelet energy (or the probability distribution), the wavelet entropies are obtained using the definition of entropy given by Shannon.

Non-linear methods: Given the complexity of the mechanisms regulating heart rate, it is reasonable to assume that applying HRV analysis based on methods of non-linear dynamics will yield valuable information. Although chaotic behavior has been assumed, more rigorous testing has shown that heart rate variability cannot be described as a chaotic process [11]. The most commonly used non-linear method of heart rate variability analysis is the Poincaré plot. Each data point represents a pair of successive beats; the $\mathrm{x}$-axis is the current RR interval, while the $y$-axis is the previous $R R$ interval. HRV is quantified by fitting mathematically defined geometric shapes to the data [12]. Other methods used are the correlation dimension, nonlinear predictability [13], pointwise correlation dimension and approximate entropy [14].

Long term correlations: Sequences of RR intervals have been found to have long-term correlations [15]. However, one flaw with these analyses is their lack of goodness-of-fit statistics, i.e. values are derived that may or may not have adequate statistical rigor.

\section{RESULTS}

We have used an ECG database from physonet of 30 subjects: 15 patients of Heart rate variability and other 15 normal subjects. The HRV database is divided into the training lot and the test lot. We have considered that the significant information for HRV analysis is concentrated on the QRST zone of the lead V5 only. The selected QRST zone of the prototype has been normalized to 8 bit sample $(m=128)$ as in Fig. 2

The Discrete Cosine Transform (DCT) applied for feature extraction has the advantage of reducing the computational effort by using algorithms methods [16]. The simulation results given in Table III show that one can reduce the space dimension from 8 bit sample $(m=128)$ to 5 bit sample $(m=32)$ using DCT, by preserving $90 \%$ of the signal energy.

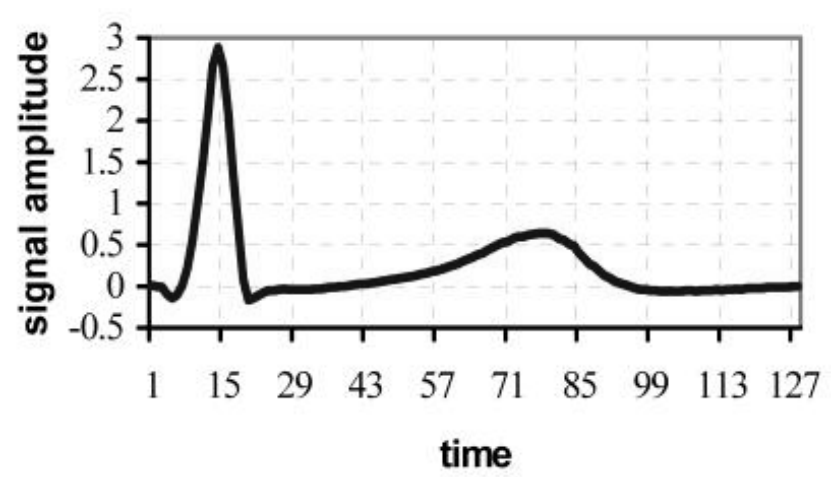

Fig. 2. ECG-QRST prototype corresponding to a HRV subject.

TABLE III: RECOGNITION SCORE OF THE NEURO-FUZZY SYSTEM ClassifIER AS A FUNCTION OF THE NUMBER OF FEATURES M.

\begin{tabular}{lcccc}
\hline \hline \multicolumn{1}{c}{ Data } & \multicolumn{4}{c}{ Number of sample m } \\
\hline $\begin{array}{l}\text { Number of retained } \\
\text { principal components } \mathrm{m}\end{array}$ & 16 & 32 & 48 & 64 \\
$\begin{array}{l}\text { Type of feature } \\
\text { extraction }\end{array}$ & DTC & DTC & DTC & DTC \\
$\begin{array}{l}\text { Recognition score for } \\
\text { the training lot (\%) }\end{array}$ & 95 & 100 & 100 & 100 \\
$\begin{array}{l}\text { Recognition score for } \\
\text { the test lot (\%) } \\
\text { Number of training } \\
\text { epochs }\end{array}$ & 85 & 90 & 94 & 95 \\
\hline \hline
\end{tabular}

Examples of DCT are given in Fig. 3.

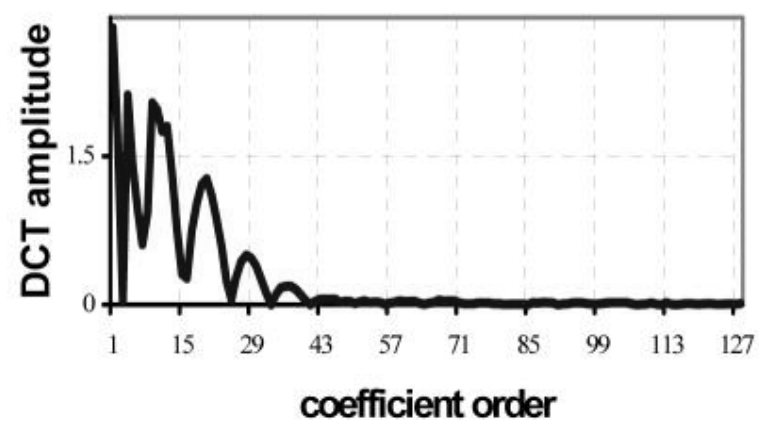

Fig. 3. Examples of DCT (amplitude spectrum).

\section{CONCLUSIONS}

This paper presents an ECG classification approach for HRV analysis using a neuro-fuzzy system. The HRV processing by extraction using either DCT method or classification using neuro-fuzzy system.

If one chooses the DCT for the same space dimensionality reduction, the energy preservation ratio decreases to $90 \%$ as in Table III. For the same number of retained features " $m$ ", the DCT usually leads to a less recognition (for example, for $m=48$, one obtains a recognition score of $94 \%$ for DCT; for $m=32$, one obtains a recognition score of $90 \%$ for DCT).

Usually, by increasing the number of retained features " $m$ ", 
the recognition score increases. This is important to scope HRV test lost signals.

\section{REFERENCES}

[1] V. E. Neagoe and I. F. Iatan, "Face recognition using a fuzzy-Gaussian neural network," in Proc First IEEE Internat. Conference on Cognitive Informatics (ICCI 2002), Calgary, August 19-20, 2002, pp. 361-368.

[2] C. D. Nugent, J. A. C. Webb, N. D. Black, G. T. H. Wright, and M McIntyre, "An intelligent framework for the classification of the 12-Lead ECG," Artificial Intelligence in Medicine, vol. 16, pp. 205-222, 1999

[3] G. Bortolan, C. Brohet, and S. Fusaro, 'Possibilities of using neural networks for ECG classification," J Electrocardiol, vol. 29 (Suppl), pp. $10-16,1996$

[4] J. E. Mietus, C. K. Peng, I. Henry, R. L. Goldsmith, and A. L. Goldberger, "The pNNx-files: Reexamining a widely-used heart rate variability measure," Heart, vol. 88, pp. 378-380, 2002.

[5] S. Sivathasan, F. Cecelja, and W. Balachandran, "ECG diagnosis using neural networks and fuzzy expert system," in Proc 17th IEEE Instrumentation and Measurement Technology Conference (IMTC 2000), vol. 2, 2000, pp. 988-992.

[6] V. E. Neagoe and I. F. Iatan, "Face recognition using a fuzzy-Gaussian neural network," in Proc First IEEE Internat, Conference on Cognitive Informatics (ICCI 2002), Calgary, August 19-20, 2002, pp. 361-368.

[7] Y. C. Chen and C. C. Teng, "Fuzzy neural network in model reference control systems," Fuzzy Logic and Expert Systems Applications, San Diego-Toronto: Academic Press, pp. 285-313, 1998.

[8] J. Jang, C. Sun, and E. Mizutani, Neuro-Fuzzy and Soft Computing, pp. 34, 208-210, 335-368, Prentice Hall, 1997.

[9] V. Neagoe and O. Stanasila, Recunoasterea formelor si retele neurale [Pattern recognition and neural networks, Ro, Bucharest: Matrix-Rom Publuishers, 1999.

[10] J. E. Mietus, C. K. Peng, I. Henry, R. L. Goldsmith, and A. L. Goldberger, "The pNNx files: re-examining a widely used heart rate variability measure," Heart, vol. 88, pp. 378-380, 2002.

[11] I. Yalcin and M. Kuntalp, "Combining classical HRV indices with wavelet entropy measures improves to performance in diagnosing congestive heart failure," Computers in Biology and Medicine, vol. 37, no. 10 , pp. $1502-1510,2007$.

[12] J. K. Kanters, N. H. Holstein-Rathlou, and E. Agner, "Lack of evidence for low-dimensional chaos in heart rate variability," Journal of Cardiovascular Electrophysiology, vol. 5, no. 7, pp. 591-601, 1994.

[13] M. Brennan, M. Palaniswami, and P. Kamen, "Do existing measures of Poincaré plot geometry reflect non-linear features of heart rate variability?" Biomedical Engineering, IEEE Transactions on, in Proc. IEEE Transactions on Biomedical Engineering, vol. 48, 2001, pp. 1342-1347.
[14] R. J. Storella et al., "Approximate entropy and point correlation dimension of heart rate variability in healthy subjects," Integrative Physiological \& Behavioral Science, vol. 33, no. 4, pp. 315-20, 1994.

[15] C. K. Peng et al., "Long-range anticorrelations and non-gaussian behavior of the heartbeat," Phys. Rev. Lett, vol. 70, no. 9, pp. 1343-6, 1993.

[16] B. G. Sherlock and D. M. Monro, "Algorithm 749: Fast discrete cosine transform," ACM Trans. on Mathematical Software (TOMS), vol. 21, no. 4, pp. 372-378, 1995.

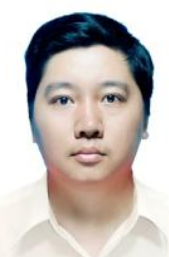

Hoang ChuDuc is a researcher at Department of Electronics and Biomedical Engineering, School of Electronics and Telecommunication, Hanoi University of Science and Technology.

He has 8 years' experience at researcher in biomedical equipment design, signal processing and teaching. His key qualifications (relevant to the project): Analysis, design, implementation of info- Ramation systems and management for hospitals and clinics; Quality management for software processing; Coordinating projects and planning medical equipment; Supervision for IT projects; Preparing bidding documents, bid evaluation; Project management and coordination for such domestic agencies as FPT, Wiettel, Panasonic, Department of IT, Ministry of Health, CMC, IBM, Oracle, hospital as well as foreign partners from Singapore, Janpan, Australia, Malaysia. From 2010, he does PhD research with project "Impact analysis between the ECG signal and the common defense of the Vietnamese."

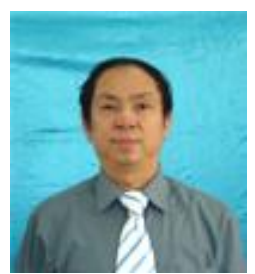

Thuan NguyenDuc is a lecture at Department of Electronics and Biomedical Engineering, School of Electronics and Telecommunication, Hanoi University of Science and Technology. He has over 30 years' experience inbiomedical equipment design, signal processing and teaching.

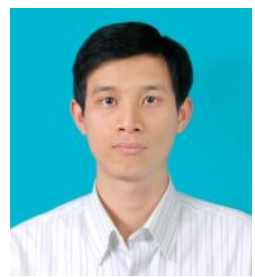

Trung LaiHuuPhuong is a researcher at Center of Biomedical Electronics, Hanoi University of Science and Technology. He has over 10 years' experience in biomedical equipment design, signal processing and teaching. 